\title{
Lie Symmetry Analysis and New Exact Solutions for a Higher-Dimensional Shallow Water Wave Equation
}

\author{
Yinghui He \\ Department of Mathematics, Honghe University, Mengzi, Yunnan 661100, China \\ Correspondence should be addressed to Yinghui He; heyinghui07@163.com
}

Received 10 May 2015; Accepted 21 June 2015

Academic Editor: Chaudry Masood Khalique

Copyright ( 2015 Yinghui He. This is an open access article distributed under the Creative Commons Attribution License, which permits unrestricted use, distribution, and reproduction in any medium, provided the original work is properly cited.

In our work, a higher-dimensional shallow water wave equation, which can be reduced to the potential KdV equation, is discussed. By using the Lie symmetry analysis, all of the geometric vector fields of the equation are obtained; the symmetry reductions are also presented. Some new nonlinear wave solutions, involving differentiable arbitrary functions, expressed by Jacobi elliptic function, Weierstrass elliptic function, hyperbolic function, and trigonometric function are obtained. Our work extends pioneer results.

\section{Introduction}

It has recently become more interesting to obtain exact solutions of nonlinear partial differential equations. These equations are mathematical models of complex physical phenomena that arise in engineering, applied mathematics, chemistry, biology, mechanics, physics, and so forth. Thus, the investigation of the traveling wave solutions to nonlinear evolution equations (NLEEs) plays an important role in mathematical physics. A lot of physical models have supported a wide variety of solitary wave solutions.

In the recent years, much efforts have been spent on finding traveling wave solution and many significant methods have been established. However, the study on nonlinear wave solution is few and there is no unified approach. In this work, we studied the nonlinear wave solution of a higher-dimensional shallow water wave equation by using Lie symmetry analysis [1-5] and extend F-expansion method [69].

Wazwaz [10] introduced the following (3+1)-dimensional equation:

$$
u_{y z t}+u_{x x y z}-6 u_{x} u_{x y z}-6 u_{x y} u_{x z}=0,
$$

as a higher-dimensional shallow water wave equation. It is easy to see that (1) can be reduced to the potential KdV equation for $z=y=x$.
In [10], Wazwaz investigated multiple soliton solutions and multiple singular soliton solutions of (1) and pointed that this equation is a completely integrable equation. In [11], Chen and Liu obtained general multiple soliton solutions and some nonlinear wave solutions of (1) by simplified Hirota's method [12,13] and Dynamical system approach [14, 15]. The main purpose of this paper is to investigate the vector fields, the symmetry reductions, and exact solutions to (1) by means of the combination of Lie symmetry analysis and the extended F-expansion method.

The rest of this paper is organized as follows. In Section 2, the Lie symmetry analysis is performed on (1); the complete geometric vector fields of the equation are obtained. In Section 3, different types of symmetry reductions of (1) are obtained. In Section 4, some new exact explicit solutions are presented. Section 5 is a short summary and discussion.

\section{Lie Symmetries for (1)}

First of all, let us consider a one-parameter Lie group of infinitesimal transformation:

$$
\begin{aligned}
& x \longrightarrow x+\epsilon \xi(x, y, z, t, u), \\
& y \longrightarrow y+\epsilon \eta(x, y, z, t, u),
\end{aligned}
$$




$$
\begin{gathered}
z \longrightarrow z+\epsilon \mu(x, y, z, t, u), \\
t \longrightarrow t+\epsilon \tau(x, y, z, t, u), \\
u \longrightarrow u+\epsilon \phi(x, y, z, t, u),
\end{gathered}
$$

with a small parameter $\epsilon \ll 1$. The vector field associated with the above group of transformations can be written as

$$
\begin{aligned}
V= & \xi(x, y, z, t, u) \frac{\partial}{\partial x}+\eta(x, y, z, t, u) \frac{\partial}{\partial y} \\
& +\mu(x, y, z, t, u) \frac{\partial}{\partial z}+\tau(x, y, z, t, u) \frac{\partial}{\partial t} \\
& +\phi(x, y, z, t, u) \frac{\partial}{\partial u} .
\end{aligned}
$$

The symmetry group of (1) will be generated by the vector field of the form (3). Applying the fourth prolongation $\mathrm{pr}^{(5)} \mathrm{V}$ to (1), we find that the coefficient functions $\xi, \eta, \mu, \tau$, and $\phi$ must satisfy the symmetry condition

$$
\begin{aligned}
& -6 u_{x y z} \phi^{x}+6\left(u_{x z} \phi^{x y}+u_{x y} \phi^{x z}\right)+\phi^{y z t}-6 u_{x} \phi^{x y z} \\
& +\phi^{x x x y z}=0,
\end{aligned}
$$

where $\phi^{x}, \phi^{x y}, \phi^{x z}, \phi^{x y z}, \phi^{y z t}$, and $\phi^{x x x y z}$ are the coefficients of $\mathrm{pr}^{(5)} V$. Furthermore, we have

$$
\begin{aligned}
\phi^{x}= & D_{x}\left(\phi-\xi u_{x}-\eta u_{y}-\mu u_{z}-\tau u_{t}\right)+\xi u_{x x} \\
& +\eta u_{x y}+\mu u_{x z}+\tau u_{x t}, \\
\phi^{x y}= & D_{y} D_{x}\left(\phi-\xi u_{x}-\eta u_{y}-\mu u_{z}-\tau u_{t}\right)+\xi u_{x x y} \\
& +\eta u_{x y y}+\mu u_{x y z}+\tau u_{x y t}, \\
\phi^{x z}= & D_{z} D_{x}\left(\phi-\xi u_{x}-\eta u_{y}-\mu u_{z}-\tau u_{t}\right)+\xi u_{x x z} \\
& +\eta u_{x y z}+\mu u_{x z z}+\tau u_{x z t}, \\
\phi^{x y z}= & D_{z} D_{y} D_{x}\left(\phi-\xi u_{x}-\eta u_{y}-\mu u_{z}-\tau u_{t}\right) \\
& +\xi u_{x x y z}+\eta u_{x y y z}+\mu u_{x y z z}+\tau u_{x y z t}, \\
& +\tau u_{x x x y z t}, \\
\phi^{y z t}= & D_{t} D_{z} D_{y}\left(\phi-\xi u_{x}-\eta u_{y}-\mu u_{z}-\tau u_{t}\right) \\
& +\xi u_{x y z t}+\eta u_{y y z t}+\mu u_{y z z t}+\tau u_{y z t t}, \\
\phi^{x x x y z}= & D_{z} D_{y} D_{x}^{3}\left(\phi-\xi u_{x}-\eta u_{y}-\mu u_{z}-\tau u_{t}\right) \\
& +\xi u_{x x x x y z}+\eta u_{x x x y y z}+\mu u_{x x x y z z} \\
&
\end{aligned}
$$

where $D_{x}^{3}=D_{x} D_{x} D_{x}, D_{x}, D_{y}, D_{z}$, and $D_{t}$ are the total derivatives with respect to $x, y, z$, and $t$, respectively.
Substituting (5) into (4), combined with (1) and equating the coefficients of the various monomials in the first, second, third, and the other partial derivatives and various powers of $u$, we can find the determining equations for the symmetry group of (1); then standard symmetry group calculations lead to the following forms of the coefficient functions:

$$
\begin{aligned}
\xi= & \frac{1}{3} c_{1} x+F_{3}(t), \\
\eta= & F_{2}(y), \\
\mu= & F_{1}(z), \\
\tau= & c_{1} t+c_{2}, \\
\phi= & -\frac{1}{3} c_{1} u-\frac{1}{6} x F_{3}^{\prime}(t)+F_{4}(z, t)+F_{5}(y, t) \\
& +F_{6}(y, z),
\end{aligned}
$$

where $F_{1}(z), F_{2}(y), F_{3}(t), F_{4}(z, t), F_{5}(y, t)$, and $F_{6}(y, z)$ are arbitrary functions on their variables; $c_{1}$ and $c_{2}$ are arbitrary constants.

Thus, in terms of the Lie symmetry analysis method, we obtain all of the geometric vector fields of (1) as follows:

$$
\begin{aligned}
V_{1}\left(F_{1}\right) & =F_{1}(z) \frac{\partial}{\partial z}, \\
V_{2}\left(F_{2}\right) & =F_{2}(y) \frac{\partial}{\partial y}, \\
V_{3}\left(F_{3}\right) & =F_{3}(t) \frac{\partial}{\partial x}-\frac{x}{6} F_{3}^{\prime}(t) \frac{\partial}{\partial u}, \\
V_{4}\left(F_{4}\right) & =F_{4}(z, t) \frac{\partial}{\partial u}, \\
V_{5}\left(F_{5}\right) & =F_{5}(y, t) \frac{\partial}{\partial u}, \\
V_{6}\left(F_{6}\right) & =F_{6}(y, z) \frac{\partial}{\partial u}, \\
V_{7} & =\frac{x}{3} \frac{\partial}{\partial x}+t \frac{\partial}{\partial t}-\frac{u}{3} \frac{\partial}{\partial u}, \\
V_{8} & =\frac{\partial}{\partial t} \cdot
\end{aligned}
$$

The symmetry of (3) can be written as

$$
\begin{aligned}
V= & V_{1}\left(F_{1}\right)+V_{2}\left(F_{2}\right)+V_{3}\left(F_{3}\right)+V_{4}\left(F_{4}\right)+V_{5}\left(F_{5}\right) \\
& +V_{6}\left(F_{6}\right)+V_{7}+V_{8} .
\end{aligned}
$$


It is necessary to check that $\left\{V_{1}\left(F_{1}\right), V_{2}\left(F_{2}\right), V_{3}\left(F_{3}\right), V_{4}\left(F_{4}\right)\right.$, $\left.V_{5}\left(F_{5}\right), V_{6}\left(F_{6}\right), V_{7}, V_{8}\right\}$ is closed under the Lie bracket. In fact, we have

$$
\begin{aligned}
& {\left[V_{i}, V_{i}\right]=0, \quad i=1,2, \ldots, 8,} \\
& {\left[V_{1}, V_{2}\right]=\left[V_{1}, V_{3}\right]=\left[V_{1}, V_{5}\right]=\left[V_{1}, V_{7}\right]=\left[V_{1}, V_{8}\right]} \\
& =0 \text {, } \\
& {\left[V_{1}, V_{4}\right]=V_{4}\left[-\left(F_{4}\right)_{t} F_{1}\right],} \\
& {\left[V_{1}, V_{6}\right]=V_{6}\left[-\left(F_{6}\right)_{z} F_{1}\right] \text {, }} \\
& {\left[V_{2}, V_{3}\right]=\left[V_{2}, V_{4}\right]=\left[V_{2}, V_{7}\right]=\left[V_{2}, V_{8}\right]=0,} \\
& {\left[V_{2}, V_{5}\right]=V_{5}\left[-\left(F_{5}\right)_{y} F_{2}\right] \text {, }} \\
& {\left[V_{2}, V_{6}\right]=V_{6}\left[-\left(F_{6}\right)_{y} F_{2}\right] \text {, }} \\
& {\left[V_{3}, V_{4}\right]=\left[V_{3}, V_{5}\right]=\left[V_{3}, V_{6}\right]=0,} \\
& {\left[V_{3}, V_{7}\right]=V_{3}\left[t F_{3}^{\prime}-\frac{F_{3}}{3}\right] \text {, }} \\
& {\left[V_{3}, V_{8}\right]=V_{3}\left[F_{3}^{\prime}\right] \text {, }} \\
& {\left[V_{4}, V_{5}\right]=\left[V_{4}, V_{6}\right]=0 \text {, }} \\
& {\left[V_{4}, V_{7}\right]=V_{4}\left[t\left(F_{4}\right)_{t}+\frac{F_{4}}{3}\right] \text {, }} \\
& {\left[V_{4}, V_{8}\right]=V_{4}\left[F_{4}\right] \text {, }} \\
& {\left[V_{5}, V_{6}\right]=0 \text {, }} \\
& {\left[V_{5}, V_{7}\right]=V_{5}\left[t\left(F_{5}\right)_{t}+\frac{F_{5}}{3}\right],} \\
& {\left[V_{5}, V_{8}\right]=V_{5}\left[F_{5}\right] \text {, }} \\
& {\left[V_{6}, V_{7}\right]=\frac{1}{3} V_{6}\left[F_{6}\right] \text {, }} \\
& {\left[V_{6}, V_{8}\right]=0 \text {, }} \\
& {\left[V_{7}, V_{8}\right]=V_{8} \text {. }}
\end{aligned}
$$

Thus, the Lie algebra of infinitesimal symmetries of (1) is spanned by the above eight vector fields (7), and (7) form a basis for the Lie algebra. The commutator table is given by the above commutation relations.

\section{Symmetry Reductions}

In this section we will obtain symmetry reductions of (1) by means of the symmetry analysis. Based on the infinitesimals (6), the similarity variables are found by solving the corresponding characteristic equations

$$
\frac{d x}{\xi}=\frac{d y}{\eta}=\frac{d z}{\mu}=\frac{d t}{\tau}=\frac{d u}{\phi}
$$

or the invariant surface conditions

$$
\begin{aligned}
\Phi= & \xi \frac{\partial}{\partial x} u(x, y, z, t)+\eta \frac{\partial}{\partial y} u(x, y, z, t) \\
& +\mu \frac{\partial}{\partial z} u(x, y, z, t)+\tau \frac{\partial}{\partial t} u(x, y, z, t)-\phi .
\end{aligned}
$$

While solving the above invariant surface conditions, one has to distinguish between cases in which some of the functions $F_{1}(z), F_{2}(y), F_{3}(t), F_{4}(z, t), F_{5}(y, t), F_{6}(y, z)$, and $c_{1}, c_{2}$ are identical to zero and cases where they are not. This leads to different relations between the similarity variables $(X, Y, Z, U)$ and the original variables $(x, y, z, t, u)$. As a result, we obtain the following cases.

Case 1. Let $c_{2}=F_{3}(t)=1$ and $c_{1}=F_{1}(z)=F_{2}(y)=F_{4}(z, t)=$ $F_{5}(y, t)=F_{6}(y, z)=0$; then

$$
\Phi=u_{t}+u_{x}
$$

Solving the differential equation $\Phi=0$ one can get

$$
u=U(X, y, z), \quad X=-x+t
$$

Substituting (13) into (1), we can reduce it to

$$
U_{X y z}+U_{X X X y z}-6 U_{X} U_{X y z}-6 U_{X y} U_{X z}=0 .
$$

Case 2. Let $c_{2}=1$ and $c_{1}=F_{1}(z)=F_{3}(t)=F_{4}(z, t)=$ $F_{5}(y, t)=F_{6}(y, z)=0$; then

$$
\Phi=u_{t}+F_{2}(y) u_{y}
$$

Solving the differential equation $\Phi=0$ one can get

$$
u=U(x, Y, z), \quad Y=-\int \frac{1}{F_{2}(y)} d y+t .
$$

Substituting (16) into (1), we can reduce it to

$$
6 U_{x} U_{x Y z}+6 U_{Y z} U_{x z}-U_{Y Y z}-U_{x x x Y z}=0 .
$$

Case 3. Let $c_{2}=1$ and $c_{1}=F_{2}(y)=F_{3}(t)=F_{4}(z, t)=$ $F_{5}(y, t)=F_{6}(y, z)=0$; then

$$
\Phi=u_{t}+F_{1}(z) u_{z}
$$

Solving the differential equation $\Phi=0$ one can get

$$
u=U(x, y, Z), \quad Z=-\int \frac{1}{F_{1}(z)} d z+t
$$

Substituting (19) into (1), we can reduce it to

$$
6 U_{x} U_{x y Z}+6 U_{x y} U_{x Z}-U_{y z Z}-U_{x x x y Z}=0 .
$$


Case 4. Let $c_{1}=c_{2}=F_{3}(t)=F_{4}(z, t)=F_{5}(y, t)=F_{6}(y, z)=0$; then

$$
\Phi=F_{2}(y) u_{y}+F_{1}(z) u_{z}
$$

Solving the differential equation $\Phi=0$ one can get

$$
u=U(x, Y, t), \quad Y=-\int \frac{1}{F_{2}(y)} d y+\int \frac{1}{F_{1}(z)} d z
$$

Substituting (22) into (1), we can reduce it to

$$
6 U_{x} U_{x Y Y}+6 U_{x Y}^{2}-U_{Y Y t}-U_{x x x Y Y}=0
$$

Case 5. Let $c_{1}=c_{2}=F_{4}(z, t)=F_{5}(y, t)=F_{6}(y, z)=0$; then

$$
\Phi=F_{2}(y) u_{y}+F_{1}(z) u_{z}+u_{x} F_{3}(t)+\frac{1}{6} x F_{3}^{\prime}(t) .
$$

Solving the differential equation $\Phi=0$ one can get

$$
\begin{aligned}
& u=-\frac{1}{12} F_{3}(t) F_{3}^{\prime}(t)\left(\int \frac{1}{F_{2}(y)} d y\right)^{2}+\frac{1}{6} x F_{3}^{\prime}(t) \int \frac{1}{F_{2}(y)} d y \\
& +U(X, Y, t) \\
& X=-F_{3}(t) \int \frac{1}{F_{2}(y)} d y+x, Y=-\int \frac{1}{F_{2}(y)} d y+\int \frac{1}{F_{1}(z)} d z .
\end{aligned}
$$

Substituting (25) into (1), we can reduce it to

$$
\begin{aligned}
& \left(6 U_{x} U_{X X Y}+6 U_{X X} U_{X Y}-U_{X X X X Y}\right) F_{3}(t) \\
& \quad+6 U_{X Y Y} U_{x}+6 U_{X Y}^{2}-U_{Y Y t}-U_{X X X Y Y}=0 .
\end{aligned}
$$

Obviously, if $F_{3}(t)=0,(26)$ becomes (23).

Case 6. Let $c_{1}=c_{2}=F_{1}(z)=F_{2}(y)=F_{5}(y, t)=F_{6}(y, z)=0$; then

$$
\Phi=F_{3}(t) u_{x}+\frac{1}{6} x F_{3}^{\prime}(t)-F_{4}(z, t)
$$

Solving the differential equation $\Phi=0$ one can get

$$
u=\frac{-x^{2} F_{3}^{\prime}(t)+12 x F_{4}(z, t)}{12 F_{3}(t)}+U(y, z, t) .
$$

Substituting (28) into (1), we can reduce it to

$$
U_{y z t}=0
$$

Its solution is $U(y, z, t)=r_{1}(y, z)+r_{2}(y, t)+r_{3}(z, t)$. So, we can obtain solution of (1) as follows:

$$
\begin{aligned}
u= & \frac{-x^{2} F_{3}^{\prime}(t)+12 x F_{4}(z, t)}{12 F_{3}(t)}+r_{1}(y, z)+r_{2}(y, t) \\
& +r_{3}(z, t),
\end{aligned}
$$

where $r_{1}(y, z), r_{2}(y, t)$, and $r_{3}(z, t)$ are arbitrary functions on their variables. Similarly, when $c_{1}=c_{2}=F_{1}(z)=F_{2}(y)=$ $F_{4}(z, t)=F_{6}(y, z)=0$, we can get the following solutions:

$$
\begin{aligned}
u= & \frac{-x^{2} F_{3}^{\prime}(t)+12 x F_{5}(y, t)}{12 F_{3}(t)}+r_{1}(y, z)+r_{2}(y, t) \\
& +r_{3}(z, t) .
\end{aligned}
$$

Case 7. Let $c_{1}=F_{3}(t)=F_{4}(z, t)=F_{5}(y, t)=F_{6}(y, z)=0$, $c_{2}=1$; then

$$
\Phi=F_{2}(y) u_{y}+F_{1}(z) u_{z}+u_{t} .
$$

Solving the differential equation $\Phi=0$ one can get

$$
\begin{aligned}
& u=U(x, Y, Z) \\
& Y=t-\int \frac{1}{F_{2}(y)} d y, Z=-\int \frac{1}{F_{2}(y)} d y+\int \frac{1}{F_{1}(z)} d z .
\end{aligned}
$$

Substituting (33) into (1), we can reduce it to

$$
\begin{gathered}
6 U_{x} U_{x Z Z}+6 U_{x} U_{x Y Z}+6 U_{x Z}^{2}+6 U_{x Y} U_{x Z}-U_{Y Z Z} \\
-U_{Y Y Z}-U_{x x x Z Z}-u_{x x x Y Z}=0 .
\end{gathered}
$$

Case 8. Let $c_{1}=c_{2}=F_{4}(z, t)=F_{5}(y, t)=F_{6}(y, z)=0$, $F_{3}(t)=1$; then

$$
\Phi=F_{2}(y) u_{y}+F_{1}(z) u_{z}+u_{x} .
$$

Solving the differential equation $\Phi=0$ one can get

$$
\begin{aligned}
& u=U(X, Y, t) \\
& X=x-\int \frac{1}{F_{2}(y)} d y, Y=-\int \frac{1}{F_{2}(y)} d y+\int \frac{1}{F_{1}(z)} d z .
\end{aligned}
$$

Substituting (36) into (1), we can reduce it to

$$
\begin{gathered}
6 U_{X} U_{X X Y}+6 U_{X} U_{X Y Y}+6 U_{X Y}^{2}+6 U_{X Y} U_{X X}-U_{X Y t} \\
-U_{Y Y t}-U_{X X X Y Y}-U_{X X X X Y}=0 .
\end{gathered}
$$

\section{The New Nonlinear Wave Solutions}

Obviously, it is easier for us to seek the explicit solutions to the reduction equations than to solve (1). The exact solutions of the reduction equations which were proposed in our work all can be solved by extended F-expansion method. By solving these reduction equations, traveling wave solutions and nontraveling wave solutions can be obtained. For simplicity, we do not discuss them in detail. In this section, by introducing a special transformation, we will give out an equivalent equation and obtain its exact solutions by extended F-expansion method.

Making a transformation

$$
u(x, y, z, t)=f(\xi)+r(y, z, t),
$$


where $\xi=k x+\psi(y, z)-c t, r(y, z, t)=r_{1}(y, z)+r_{2}(y, t)+$ $r_{3}(z, t), r_{1}(y, z), r_{2}(y, t), r_{3}(z, t)$, and $\psi(y, z)$ are arbitrary differential functions, (1) can be reduced to the following ODE:

$$
\begin{gathered}
{\left[-6 k^{2}\left(f^{\prime \prime}\right)^{2}-6 k^{2} f^{\prime} f^{\prime \prime \prime}-c f^{\prime \prime \prime}+k^{3} f^{(5)}\right] \varphi_{y} \psi_{z}} \\
+\left[-c f^{\prime \prime}-6 k^{2} f^{\prime} f^{\prime \prime}+k^{3} f^{(4)}\right] \psi_{y z}=0
\end{gathered}
$$

where $f^{\prime}=d f / d \xi, f^{\prime \prime}=d^{2} f / d \xi^{2}, \ldots$, and $k$ and $c$ are nonzero constants. $r_{1}(y, z), r_{2}(y, t), r_{3}(z, t)$, and $\psi(y, z)$ are arbitrary differential functions.

Since $\psi(y, z)$ is arbitrary, (39) holds if and only if

$$
\begin{aligned}
-6 k^{2}\left(f^{\prime \prime}\right)^{2}-6 k^{2} f^{\prime} f^{\prime \prime \prime}-c f^{\prime \prime \prime}+k^{3} f^{(5)} & =0, \\
-c f^{\prime \prime}-6 k^{2} f^{\prime} f^{\prime \prime}+k^{3} f^{(4)} & =0 .
\end{aligned}
$$

Note that (40) comes from taking the derivative on both sides of (41). Therefore we only consider (41). Integrating (41) once, it follows that

$$
f^{\prime \prime \prime}=R+\frac{c}{k^{3}} f^{\prime}+\frac{3}{k} f^{\prime 2},
$$

where $R$ is an integral constant. If $f^{\prime}(\xi)=\phi(\xi)$, then (42) becomes

$$
\phi^{\prime \prime}=R+\frac{c}{k^{3}} \phi+\frac{3}{k} \phi^{2}
$$

Balancing $\phi^{\prime \prime}$ and $\phi^{2}$ in (43), we obtain $n+2=2 n$ which gives $n=2$. Suppose that (43) owns the solutions in the form

$$
\phi(\xi)=A_{0}+A_{1} F(\xi)+A_{2} F^{2}(\xi)+\frac{B_{1}}{F(\xi)}+\frac{B_{2}}{F(\xi)^{2}},
$$

where $F(\xi)$ satisfies the following equation:

$$
\begin{aligned}
\left(F^{\prime}(\xi)\right)^{2}= & h_{0}+h_{1} F(\xi)+h_{2} F^{2}(\xi)+h_{3} F^{3}(\xi) \\
& +h_{4} F^{4}(\xi)
\end{aligned}
$$

where $h_{0}, h_{1}, h_{2}, h_{3}$, and $h_{4}$ are constant.

Substituting (44) and (45) into (43) and then setting all the coefficients of $F^{k}(k=-4, \ldots, 4)$ of the resulting system to zero, we can obtain the following results.

4.1. $h_{1}=h_{3}=0$. In this situation, we obtain the following set of nontrivial solutions:

$$
\begin{aligned}
& A_{0}=\frac{4 k^{3} h_{2}-c}{6 k^{2}}, \\
& A_{1}=0, \\
& A_{2}=0, \\
& B_{1}=0,
\end{aligned}
$$

$$
\begin{aligned}
& B_{2}=2 k h_{0}, \\
& A_{0}=\frac{4 k^{3} h_{2}-c}{6 k^{2}}, \\
& A_{1}=0, \\
& A_{2}=2 k h_{4}, \\
& B_{1}=0, \\
& B_{2}=0, \\
& A_{0}=\frac{4 k^{3} h_{2}-c}{6 k^{2}}, \\
& A_{1}=0, \\
& A_{2}=2 k h_{4}, \\
& B_{1}=0, \\
& B_{2}=2 k h_{0},
\end{aligned}
$$

where $h_{0}, h_{2}$, and $h_{4}$ are arbitrary constants and $k$ and $c$ are nonzero constants.

Substituting (46) into (44), we obtain, respectively, the following solutions of (43):

$$
\begin{aligned}
& \phi(\xi)=\frac{4 k^{3} h_{2}-c}{6 k^{2}}+\frac{2 k h_{0}}{F^{2}(\xi)}, \\
& \phi(\xi)=\frac{4 k^{3} h_{2}-c}{6 k^{2}}+2 k h_{4} F^{2}(\xi), \\
& \phi(\xi)=\frac{4 k^{3} h_{2}-c}{6 k^{2}}+\frac{2 k h_{0}}{F^{2}(\xi)}+2 k h_{4} F^{2}(\xi),
\end{aligned}
$$

where $\xi=k x+\psi(y, z)-c t$ and $r(y, z, t)=r_{1}(y, z)+r_{2}(y, t)+$ $r_{3}(z, t)$.

When $h_{1}=h_{3}=0$, the general elliptic equation (45) is reduced to the auxiliary ordinary equation

$$
F^{\prime}(\xi)^{2}=h_{0}+h_{2} F^{2}(\xi)+h_{4} F^{4}(\xi) .
$$

The solutions of (50) are given in Table 1. Combining (47)-(49) with Table 1, many exact solutions of (43) can be obtained. For simplicity, we just give out the first case in Table 1; the other cases can be discussed similarly.

When $h_{0}=1, h_{2}=-\left(m^{2}+1\right)$, and $h_{4}=m^{2}$, the solution of (50) is $F(\xi)=\operatorname{sn}(\xi, m)$ or $F(\xi)=\operatorname{cd}(\xi, m)$. Substituting them into (47)-(49), we can obtain the following Jacobi Elliptic function solutions of (1).

From (47), one has

$$
\begin{aligned}
& f^{\prime}(\xi)=\phi(\xi)=-\frac{4 k^{3} m^{2}+4 k^{3}+c}{6 k^{2}}+2 k \mathrm{~ns}^{2}(\xi, m), \\
& f^{\prime}(\xi)=\phi(\xi)=-\frac{4 k^{3} m^{2}+4 k^{3}+c}{6 k^{2}}+2 k \mathrm{dc}^{2}(\xi, m) .
\end{aligned}
$$


TABLE 1: Solutions of $F(\xi)$ in $F^{\prime 2}=h_{0}+h_{2} F^{2}+h_{4} F^{4}$.

\begin{tabular}{lcccc}
\hline Case & $h_{0}$ & $h_{2}$ & $h_{4}$ & $F(\xi)$ \\
\hline 1 & 1 & $-\left(m^{2}+1\right)$ & $m^{2}$ & $\operatorname{sn}(\xi), \mathrm{cd}(\xi)$ \\
2 & $1-m^{2}$ & $m^{2}-1$ & $-m^{2}$ & $\mathrm{cn}(\xi)$ \\
3 & $m^{2}-1$ & $2-m^{2}$ & -1 & $\mathrm{dn}(\xi)$ \\
4 & $m^{2}$ & $-\left(m^{2}+1\right)$ & 1 & $\mathrm{~ns}(\xi), \mathrm{dc}(\xi)$ \\
5 & $-m^{2}$ & $2 m^{2}-1$ & $1-m^{2}$ & $\mathrm{nc}(\xi)$ \\
6 & -1 & $2-m^{2}$ & $m^{2}-1$ & $\mathrm{nd}(\xi)$ \\
7 & 1 & $2-m^{2}$ & $1-m^{2}$ & $\operatorname{sc}(\xi)$ \\
8 & 1 & $2 m^{2}-1$ & $-m^{2}\left(1-m^{2}\right)$ & $\operatorname{sd}(\xi)$ \\
9 & $1-m^{2}$ & $2-m^{2}$ & 1 & $\operatorname{cs}(\xi)$ \\
10 & $-m^{2}\left(1-m^{2}\right)$ & $2 m^{2}-1$ & 1 & $\operatorname{sd}(\xi)$ \\
11 & $\frac{1}{4}$ & $\frac{1-2 m^{2}}{2}$ & $\frac{1}{4}$ & $\mathrm{~ns}(\xi) \pm \mathrm{cs}(\xi)$ \\
12 & $\frac{1-m^{2}}{4}$ & $\frac{1+m^{2}}{2}$ & $\frac{1-m^{2}}{4}$ & $\mathrm{nc}(\xi) \pm \mathrm{sc}(\xi)$ \\
13 & $\frac{m^{2}}{4}$ & $\frac{m^{2}-2}{2}$ & $\frac{1}{4}$ & $\mathrm{~ns}(\xi) \pm \mathrm{ds}(\xi)$ \\
14 & $\frac{m^{2}}{4}$ & $\frac{m^{2}-2}{2}$ & $\frac{m^{2}}{4}$ & $\operatorname{sn}(\xi) \pm i \mathrm{cn}(\xi)$ \\
\hline
\end{tabular}

Therefore, solutions of (1) can be expressed as

$$
\begin{aligned}
u(x, y, z, t)= & f(\xi)+r(y, z, t) \\
= & -\frac{4 k^{3} m^{2}-8 k^{3}+c}{6 k^{2}} \xi \\
& -2 k \text { EllipticE }(\operatorname{sn}(\xi, m), m) \\
& -2 k \frac{\mathrm{ds}(\xi, m) \mathrm{cs}(\xi, m)}{\mathrm{ns}(\xi, m)}+r(y, z, t), \\
u(x, y, z, t)= & f(\xi)+r(y, z, t) \\
= & -\frac{4 k^{3} m^{2}-8 k^{3}+c}{6 k^{2}} \xi \\
& -2 k \operatorname{EllipticE}(\operatorname{sn}(\xi, m), m) \\
& +2 k \frac{\mathrm{dc}(\xi, m) \operatorname{sc}(\xi, m)}{\operatorname{nc}(\xi, m)}+r(y, z, t) .
\end{aligned}
$$

When $m \rightarrow 1, \mathrm{~ns}(\xi, m) \rightarrow \operatorname{coth}(\xi)$, solution (51) becomes

$$
f^{\prime}(\xi)=\phi(\xi)=-\frac{8 k^{3}+c}{6 k^{2}}+2 k \operatorname{coth}^{2}(\xi) .
$$

Thus, one has

$$
\begin{aligned}
u(x, y, z, t)= & f(\xi)+r(y, z, t) \\
= & -\frac{8 k^{3}+c}{6 k^{2}} \xi \\
& -k\left(2 \operatorname{coth}(\xi)-\ln \frac{1+\operatorname{coth}(\xi)}{1-\operatorname{coth}(\xi)}\right) \\
& +r(y, z, t) .
\end{aligned}
$$

When $m \rightarrow 0$, ns $(\xi, m) \rightarrow \csc (\xi)$, solution (51) becomes

$$
f^{\prime}(\xi)=\phi(\xi)=-\frac{4 k^{3}+c}{6 k^{2}}+2 k \csc ^{2}(\xi)
$$

Thus, one has

$$
\begin{aligned}
u(x, y, z, t) & =f(\xi)+r(y, z, t) \\
& =-\frac{4 k^{3}+c}{6 k^{2}} \xi-2 k \cot (\xi)+r(y, z, t)
\end{aligned}
$$

When $m \rightarrow 0, \operatorname{dc}(\xi, m) \rightarrow \sec (\xi)$, solution (52) becomes

$$
f^{\prime}(\xi)=\phi(\xi)=-\frac{4 k^{3}+c}{6 k^{2}}+2 k \sec ^{2}(\xi) .
$$

Thus, one has

$$
\begin{aligned}
u(x, y, z, t) & =f(\xi)+r(y, z, t) \\
& =-\frac{4 k^{3}+c}{6 k^{2}} \xi+2 k \tan (\xi)+r(y, z, t)
\end{aligned}
$$

When $m \rightarrow 1, \mathrm{dc}(\xi, m) \rightarrow 1$, solution (52) becomes

$$
f^{\prime}(\xi)=\phi(\xi)=-\frac{4 k^{3}+c}{6 k^{2}} .
$$

Thus, one has

$$
\begin{aligned}
u(x, y, z, t) & =f(\xi)+r(y, z, t) \\
& =-\frac{4 k^{3}+c}{6 k^{2}} \xi+r(y, z, t) .
\end{aligned}
$$

It is easy to find that, for an arbitrary constant $K, K \xi+r(y, z, t)$ is the solution of (1).

From (48), we have

$$
\begin{aligned}
f^{\prime}(\xi) & =\phi(\xi) \\
& =-\frac{4 k^{3} m^{2}+4 k^{3}+c}{6 k^{2}}+2 k m^{2} \operatorname{sn}^{2}(\xi, m), \\
f^{\prime}(\xi) & =\phi(\xi) \\
& =-\frac{4 k^{3} m^{2}+4 k^{3}+c}{6 k^{2}}+2 k m^{2} c^{2}(\xi, m) .
\end{aligned}
$$


Therefore, solutions of (1) can be expressed as

$$
\begin{aligned}
u(x, y, z, t)= & f(\xi)+r(y, z, t) \\
= & -\frac{4 k^{3} m^{2}-8 k^{3}+c}{6 k^{2}} \xi \\
& -2 k \operatorname{EllipticE}(\operatorname{sn}(\xi, m), m) \\
& +r(y, z, t), \\
u(x, y, z, t)= & f(\xi)+r(y, z, t) \\
= & -\frac{4 k^{3} m^{2}-8 k^{3}+c}{6 k^{2}} \xi \\
& -2 k \operatorname{EllipticE}(\operatorname{sn}(\xi, m), m) \\
& -2 k m^{2} \frac{\operatorname{sc}(\xi, m) \mathrm{cd}(\xi, m)}{\operatorname{nd}(\xi, m)} \\
& +r(y, z, t) .
\end{aligned}
$$

When $m \rightarrow 1, \operatorname{sn}(\xi, m) \rightarrow \tanh (\xi)$, solution (62) becomes

$$
f^{\prime}(\xi)=\phi(\xi)=-\frac{8 k^{3}+c}{6 k^{2}}+2 k \tanh ^{2}(\xi) .
$$

Thus, one has

$$
\begin{aligned}
u(x, y, z, t)= & f(\xi)+r(y, z, t) \\
= & -\frac{8 k^{3}+c}{6 k^{2}} \xi \\
& -k\left(2 \tanh (\xi)-\ln \frac{1+\tanh (\xi)}{1-\tanh (\xi)}\right) \\
& +r(y, z, t) .
\end{aligned}
$$

From (49), we have

$$
\begin{aligned}
f^{\prime}(\xi)= & \phi(\xi) \\
= & -\frac{4 k^{3} m^{2}+4 k^{3}+c}{6 k^{2}}+2 k m^{2} \operatorname{sn}^{2}(\xi, m) \\
& +2 k \mathrm{~ns}^{2}(\xi, m), \\
f^{\prime}(\xi)= & \phi(\xi) \\
= & -\frac{4 k^{3} m^{2}+4 k^{3}+c}{6 k^{2}}+2 k m^{2} \mathrm{~cd}^{2}(\xi, m) \\
& +2 k \mathrm{dc}^{2}(\xi, m) .
\end{aligned}
$$

Therefore, solutions of (1) can be expressed as

$$
\begin{aligned}
u(x, y, z, t)= & f(\xi)+r(y, z, t) \\
= & -\frac{4 k^{3} m^{2}-20 k^{3}+c}{6 k^{2}} \xi \\
& -4 k \operatorname{EllipticE}(\operatorname{sn}(\eta, m), m) \\
& -2 k \frac{\mathrm{ds}(\xi, m) \operatorname{cs}(\xi, m)}{\mathrm{ns}(\xi, m)}+r(y, z, t),
\end{aligned}
$$

$$
\begin{aligned}
u(x, y, z, t)= & f(\xi)+r(y, z, t) \\
= & -\frac{4 k^{3} m^{2}-20 k^{3}+c}{6 k^{2}} \xi \\
& -4 k \operatorname{EllipticE}(\operatorname{sn}(\eta, m), m) \\
& +2 k \frac{\operatorname{dc}(\xi, m) \operatorname{sc}(\xi, m)}{\operatorname{nd}(\xi, m)} \\
& +2 k m^{2} \frac{\operatorname{sd}(\xi, m) \mathrm{cd}(\xi, m)}{\operatorname{nd}(\xi, m)} \\
& +r(y, z, t) .
\end{aligned}
$$

When $m \rightarrow 1, \operatorname{sn}(\xi, m) \rightarrow \tanh (\xi)$, solution (67) becomes

$$
\begin{aligned}
f^{\prime}(\xi) & =\phi(\xi) \\
& =-\frac{8 k^{3}+c}{6 k^{2}}+2 k \tanh ^{2}(\xi)+2 k \operatorname{coth}^{2}(\xi) .
\end{aligned}
$$

Thus, one has

$$
u(x, y, z, t)=f(\xi)+r(y, z, t)
$$

$$
\begin{aligned}
= & -\frac{8 k^{3}+c}{6 k^{2}} \xi-2 k(\tanh (\xi)-\operatorname{coth}(\xi)) \\
& +k\left(\ln \frac{(1+\tanh (\xi))(1+\operatorname{coth}(\xi))}{(1-\tanh (\xi))(1-\cot (\xi))}\right) \\
& +r(y, z, t) .
\end{aligned}
$$

4.2. $h_{0}=h_{4}=0$. In this situation, we have the following result:

$$
\begin{aligned}
& A_{0}=\frac{k^{3} h_{2}-c}{6 k^{2}}, \\
& A_{1}=0, \\
& A_{2}=0, \\
& B_{1}=\frac{1}{2} k h_{0}, \\
& B_{2}=0, \\
& A_{0}=\frac{k^{3} h_{2}-c}{6 k^{2}}, \\
& A_{1}=\frac{1}{2} k h_{3}, \\
& A_{2}=0, \\
& B_{1}=0, \\
& B_{2}=0, \\
& A_{0}=\frac{k^{3} h_{2}-c}{6 k^{2}},
\end{aligned}
$$




$$
\begin{aligned}
& A_{1}=\frac{1}{2} k h_{3}, \\
& A_{2}=0, \\
& B_{1}=\frac{1}{2} k h_{0}, \\
& B_{2}=0 .
\end{aligned}
$$

When $h_{0}=h_{4}=0$, the general elliptic equation (45) is reduced to the auxiliary ordinary equation

$$
F^{\prime}(\xi)^{2}=h_{1} F(\xi)+h_{2} F^{2}(\xi)+h_{3} F^{3}(\xi)
$$

Combining (72) with solutions of (73), many exact solutions of (43) can be obtained. The process is similar to the case of $h_{1}=h_{3}=0$; we omit it.

4.3. $h_{0}=h_{1}=0$. In this situation, we have the following result:

$$
\begin{aligned}
& A_{0}=\frac{k^{3} h_{2}-c}{6 k^{2}}, \\
& A_{1}=k h_{3}, \\
& A_{2}=\frac{k h_{3}^{2}}{2 h_{2}}, \\
& B_{1}=0, \\
& B_{2}=0, \\
& h_{4}=\frac{h_{3}^{2}}{4 h_{2}},
\end{aligned}
$$

where $k, c, h_{2}$, and $h_{3}$ are arbitrary constants.

Substituting (74) into (44), we obtain the following solution of (43):

$$
\phi(\xi)=\frac{k^{3} h_{2}-c}{6 k^{2}}+k h_{3} F(\xi)+\frac{k h_{3}^{2}}{2 h_{2}} F^{2}(\xi)+r(y, z, t),
$$

where $\xi=k x+\psi(y, z)-c t$ and $r(y, z, t)=r_{1}(y, z)+r_{2}(y, t)+$ $r_{3}(z, t)$.

When $h_{0}=h_{1}=0$, the general elliptic equation (45) is reduced to the auxiliary ordinary equation

$$
F^{\prime}(\xi)^{2}=h_{2} F(\xi)^{2}+h_{3} F^{3}(\xi)+h_{4} F^{4}(\xi) .
$$

If $h_{2}>0, h_{3}^{2}-4 h_{2} h_{4}=0$, the solutions of (76) are given by the following:

$$
\begin{aligned}
& F(\xi)=\frac{-h_{2}}{h_{3}}\left(1 \pm \operatorname{coth}\left(\frac{\sqrt{h_{2}}}{2} \xi+\xi_{0}\right)\right)=\frac{-h_{2}}{h_{3}} F_{1}(\xi), \\
& F(\xi)=\frac{-h_{2}}{h_{3}}\left(1 \pm \tanh \left(\frac{\sqrt{h_{2}}}{2} \xi+\xi_{0}\right)\right)=\frac{-h_{2}}{h_{3}} F_{1}(\xi),
\end{aligned}
$$

where $\xi_{0}$ is arbitrary constant.
Substituting (77) into (75), we have

$$
\begin{aligned}
f^{\prime}(\xi) & =\phi(\xi)=\frac{k^{3} h_{2}-c}{6 k^{2}}-k h_{2} F_{1}(\xi)-\frac{k h_{3}}{2} F_{1}^{2}(\xi) \\
f^{\prime}(\xi) & =\phi(\xi)=u(x, y, z, t) \\
& =\frac{k^{3} h_{2}-c}{6 k^{2}}-k h_{2} F_{2}(\xi)-\frac{k h_{3}}{2} F_{2}^{2}(\xi) .
\end{aligned}
$$

With (78) or (79), $f(\xi)$ can be solved. Then, exact solutions of (1) can be obtained.

4.4. $h_{0} \neq 0, h_{1} \neq 0, h_{2} \neq 0, h_{3} \neq 0, h_{4} \neq 0$. In this case, there exists three parameters $r, p$, and $q$ such that

$$
\begin{aligned}
\left(F^{\prime}(\xi)\right)^{2}= & h_{0}+h_{1} F(\xi)+h_{2} F^{2}(\xi)+h_{3} F^{3}(\xi) \\
& +h_{4} F^{4}(\xi)=\left(r+p F(\xi)+q F^{2}(\xi)\right)^{2} .
\end{aligned}
$$

Equation (80) is satisfied only if the following relations hold:

$$
\begin{aligned}
& h_{0}=r^{2}, \\
& h_{1}=2 r p, \\
& h_{2}=2 r q+p^{2}, \\
& h_{3}=2 p q, \\
& h_{4}=q^{2} .
\end{aligned}
$$

Equation (80) is the general Riccati equation. The solutions of (80) are listed in [9]. There are 24 group solutions named $\phi_{i}^{I},(i=1,2, \ldots, 24)$, which we do not list for simplicity.

Substituting (80) and (44) into (43) and then setting all the coefficients of $F^{k}(k=-4, \ldots, 4)$ of the resulting system to zero, we can obtain the following results:

$$
\begin{aligned}
& A_{0}=\frac{\left(p^{2}+8 q r\right) k^{3}-c}{6 k^{2}}, \\
& A_{1}=0, \\
& A_{2}=0, \\
& B_{1}=2 k p r, \\
& B_{2}=2 k r^{2}, \\
& A_{0}=\frac{\left(p^{2}+8 q r\right) k^{3}-c}{6 k^{2}}, \\
& A_{1}=2 k p q, \\
& A_{2}=2 k q^{2}, \\
& B_{1}=0, \\
& B_{2}=0,
\end{aligned}
$$

where $r, p, q, k$, and $c$ are arbitrary constants. 
Substituting (82) into (44), we obtain, respectively, the following solutions of (43):

$$
\begin{aligned}
& \phi(\xi)=\frac{\left(p^{2}+8 q r\right) k^{3}-c}{6 k^{2}}+\frac{2 k p r}{F(\xi)}+\frac{2 k r^{2}}{F^{2}(\xi)}, \\
& \phi(\xi)=\frac{\left(p^{2}+8 q r\right) k^{3}-c}{6 k^{2}}+2 k p q F(\xi)+2 k q^{2} F^{2}(\xi),
\end{aligned}
$$

where $\xi=k x+\psi(y, z)-c t$ and $r(y, z, t)=r_{1}(y, z)+r_{2}(y, t)+$ $r_{3}(z, t)$.

Substituting solutions of $(80) F(\xi)=\phi_{i}^{I}, \quad(i=1,2, \ldots, 24)$ into (83) and (84), we can obtain a lot of solutions $\phi(\xi)$ of (43). We just give one example.

When $p^{2}-4 q r>0$ and $p q \neq 0, \phi_{1}^{I}=-(1 / 2 q)(p+$ $\left.\sqrt{p^{2}-4 q r} \tanh \left(\left(\sqrt{p^{2}-4 q r} / 2\right) \xi\right)\right)$. Substituting $\phi_{1}^{I}$ into (83) and (84), we have

$$
\begin{aligned}
f^{\prime}(\xi) & =\phi(\xi)=\frac{\left(p^{2}+8 q r\right) k^{3}-c}{6 k^{2}}+\frac{2 k p r}{\phi_{1}^{I}}+\frac{2 k r^{2}}{\phi_{1}^{I^{2}}} \\
f^{\prime}(\xi) & =\phi(\xi)=u(x, y, z, t) \\
& =\frac{\left(p^{2}+8 q r\right) k^{3}-c}{6 k^{2}}+2 k p q \phi_{1}^{I}+2 k q^{2} \phi_{1}^{I^{2}} .
\end{aligned}
$$

With (85) or (86), $f(\xi)$ can be solved. Then, exact solutions of (1) can be obtained.

4.5. $h_{0} \neq 0, h_{1} \neq 0, h_{2}=0, h_{3} \neq 0, h_{4} \neq 0$. In this case, there exists three parameters $r, p$, and $q$ such that

$$
\begin{aligned}
\left(F^{\prime}(\xi)\right)^{2} & =h_{0}+h_{1} F(\xi)+h_{3} F^{3}(\xi)+h_{4} F^{4}(\xi) \\
& =\left(r+p F(\xi)+q F^{2}(\xi)\right)^{2} .
\end{aligned}
$$

Equation (87) is satisfied only if the following relations hold:

$$
\begin{aligned}
& h_{0}=r^{2}, \\
& h_{1}=2 r p, \\
& h_{3}=2 p q, \\
& h_{4}=q^{2} .
\end{aligned}
$$

The following constraint should exist between $r, p$, and $q$ parameters:

$$
p^{2}=-2 q r, \quad q r<0
$$

Therefore, we can discuss the solution of (1) similarly as Section 4.4 under the condition (89). Here, we omit it.
4.6. $h_{2}=0, h_{4}=0$. In this situation, we have the following result:

$$
\begin{aligned}
& A_{0}=-\frac{c}{6 k^{2}}, \\
& A_{1}=\frac{1}{2} k h_{3}, \\
& A_{2}=0 \\
& B_{1}=0 \\
& B_{2}=0 .
\end{aligned}
$$

Substituting (90) into (44), we obtain the following solution of (43):

$$
\phi(\xi)=-\frac{c}{6 k^{2}}+\frac{1}{2} k h_{3} F(\xi)
$$

where $\xi=k x+\psi(y, z)-c t$ and $r(y, z, t)=r_{1}(y, z)+r_{2}(y, t)+$ $r_{3}(z, t)$.

When $h_{2}=h_{4}=0$, the general elliptic equation (45) is reduced to the auxiliary ordinary equation

$$
F^{\prime}(\xi)^{2}=h_{0}+h_{1} F(\xi)+h_{3} F^{3}(\xi) .
$$

The solution of (92) is the Weierstrass elliptic doubly periodic type solution:

$$
F(\xi)=\text { WeierstrassP }\left(\frac{\sqrt{h_{3}}}{2} \xi, g_{2}, g_{3}\right), \quad h_{3}>0,
$$

where $g_{2}=-4 h_{1} / h_{3}, g_{3}=-4 h_{0} / h_{3}$, and $h_{3}>0$. Substituting (93) into (92), the solution of (43) is

$$
\begin{aligned}
f^{\prime}(\xi)= & \phi(\xi) \\
= & -\frac{c}{6 k^{2}} \\
& +\frac{1}{2} k h_{3} \text { WeierstrassP }\left(\frac{\sqrt{h_{3}}}{2} \xi, g_{2}, g_{3}\right) .
\end{aligned}
$$

Therefore, exact solutions of (1) can be expressed:

$$
\begin{aligned}
u(x, y, z, t) & =f(\xi)+r(y, z, t) \\
= & -\frac{c}{6 k^{2}} \xi \\
& -k \sqrt{h_{3}} \text { WeierstrassZeta }\left(\frac{\sqrt{h_{3}}}{2} \xi, g_{2}, g_{3}\right) \\
& +r(y, z, t) .
\end{aligned}
$$

\section{Conclusions}

In this paper, employing two methods, we studied a higherdimensional shallow water wave equation (1). Firstly, the invariance property of (1) is presented by using the Lie symmetry analysis. Then, all of the geometric vector fields and the symmetry reductions are obtained for the first 
time. Furthermore, some new nonlinear wave solutions are obtained by using a special transformation and the extended F-expansion method. The correctness of all the solutions is verified by substituting them into original equation (1). It is interesting that these solutions contain some arbitrary functions. This property seems very special. In fact, more exact explicit solutions of (1) can be derived through the reduction equations. Because of the arbitrary functions $\psi(x, y), F_{1}(z), F_{2}(y), F_{3}(t), F_{4}(z, t), F_{5}(y, t), F_{6}(y, z), r_{1}(y, z)$, $r_{2}(y, t)$, and $r_{3}(z, t)$, these solutions may be more meaningful under special conditions and may be useful to explain some physical phenomena. The method is effective to highdimensional differential equations and can also be applied to other nonlinear evolution ones.

\section{Conflict of Interests}

The author declares that there is no conflict of interests regarding the publication of this paper.

\section{Acknowledgments}

This research is supported by the Natural Science Foundation of china (nos. 11461022, 11161020, and 11361023), Science Foundation of Yunnan province (2014FA037), and Middle-Aged Academic Backbone of Honghe University (no. 2014GG0105).

\section{References}

[1] P. Olver, Applications of Lie Groups to Differential Equations, vol. 107 of Graduate Texts in Mathematics, Springer, New York, NY, USA, 1993.

[2] G. Bluman and S. Anco, Symmetry and Integration Methods for Differential Equations, vol.154 of Applied Mathematical Sciences, Springer, New York, NY, USA, 2002.

[3] H. Z. Liu and J. B. Li, "Symmetry reductions, dynamical behavior and exact explicit solutions to the Gordon types of equations," Journal of Computational and Applied Mathematics, vol. 257, pp. 144-156, 2014.

[4] H. Z. Liu and Y. X. Geng, "Symmetry reductions and exact solutions to the systems of carbon nanotubes conveying fluid," Journal of Differential Equations, vol. 254, no. 5, pp. 2289-2303, 2013.

[5] H. Z. Yang, W. Liu, B. Y. Yang, and B. He, "Lie symmetry analysis and exact explicit solutions of three-dimensional KudryashovSinelshchikov equation," Communications in Nonlinear Science and Numerical Simulation, vol. 27, no. 1-3, pp. 271-280, 2015.

[6] E. Fan, "Uniformly constructing a series of explicit exact solutions to nonlinear equations in mathematical physics," Chaos, Solitons and Fractals, vol. 16, no. 5, pp. 819-839, 2003.

[7] M. L. Wang and X. Z. Li, "Applications of F-expansion to periodic wave solutions for a new Hamiltonian amplitude equation," Chaos, Solitons and Fractals, vol. 24, no. 5, pp. 12571268, 2005.

[8] D. Wang and H.-Q. Zhang, "Further improved F-expansion method and new exact solutions of Konopelchenko-Dubrovsky equation," Chaos, Solitons \& Fractals, vol. 25, no. 3, pp. 601-610, 2005.
[9] E. Yomba, “The extended Fan's sub-equation method and its application to KdV-MKdV, BKK and variant Boussinesq equations," Physics Letters A, vol. 336, no. 6, pp. 463-476, 2005.

[10] A.-M. Wazwaz, "Multiple-soliton solutions and multiplesingular soliton solutions for two higher-dimensional shallow water wave equations," Applied Mathematics and Computation, vol. 211, no. 2, pp. 495-501, 2009.

[11] Y. R. Chen and R. Liu, "Some new nonlinear wave solutions for two (3+1)-dimensional equations," Applied Mathematics and Computation, vol. 260, pp. 397-411, 2015.

[12] R. Hirota, The Direct Method in Soliton Theory, Cambridge University Press, Cambridge, UK, 2004.

[13] R. Hirota, "Exact solutions of the korteweg-devries equation for multiple collisions of solutions," Physical Review Letters, vol. 27, no. 18, pp. 1192-1194, 1971.

[14] J. B. Li and Z. R. Liu, "Smooth and non-smooth traveling waves in a nonlinearly dispersive equation," Applied Mathematical Modelling, vol. 25, no. 1, pp. 41-56, 2000.

[15] J. B. Li and J. Lin, "Exact solutions and bifurcations of a modulated equation in a discrete nonlinear electrical transmission line (I)," International Journal of Bifurcation and Chaos, vol. 25, no. 1, Article ID 1550016, 2015. 


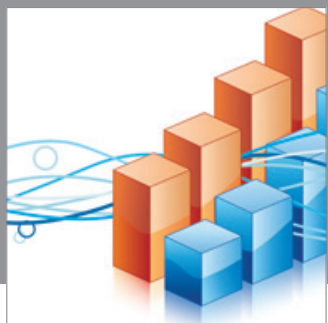

Advances in

Operations Research

mansans

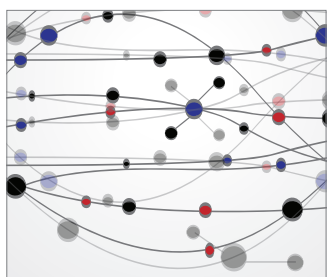

The Scientific World Journal
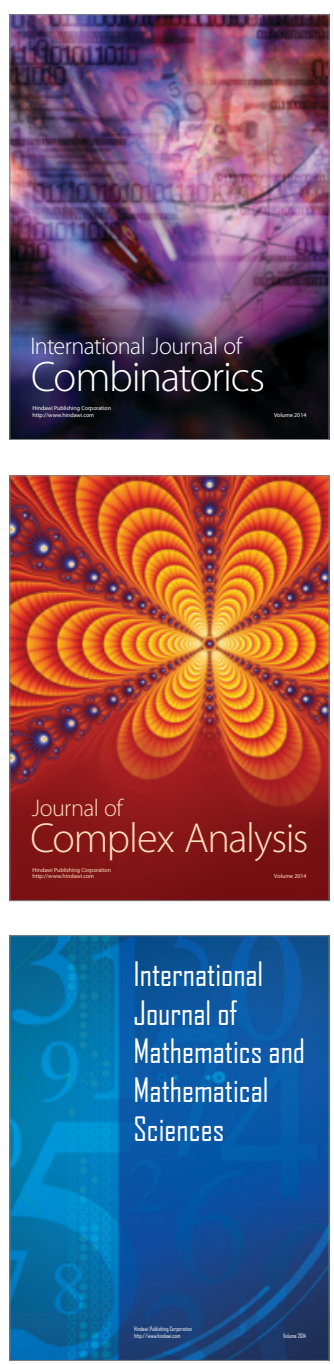
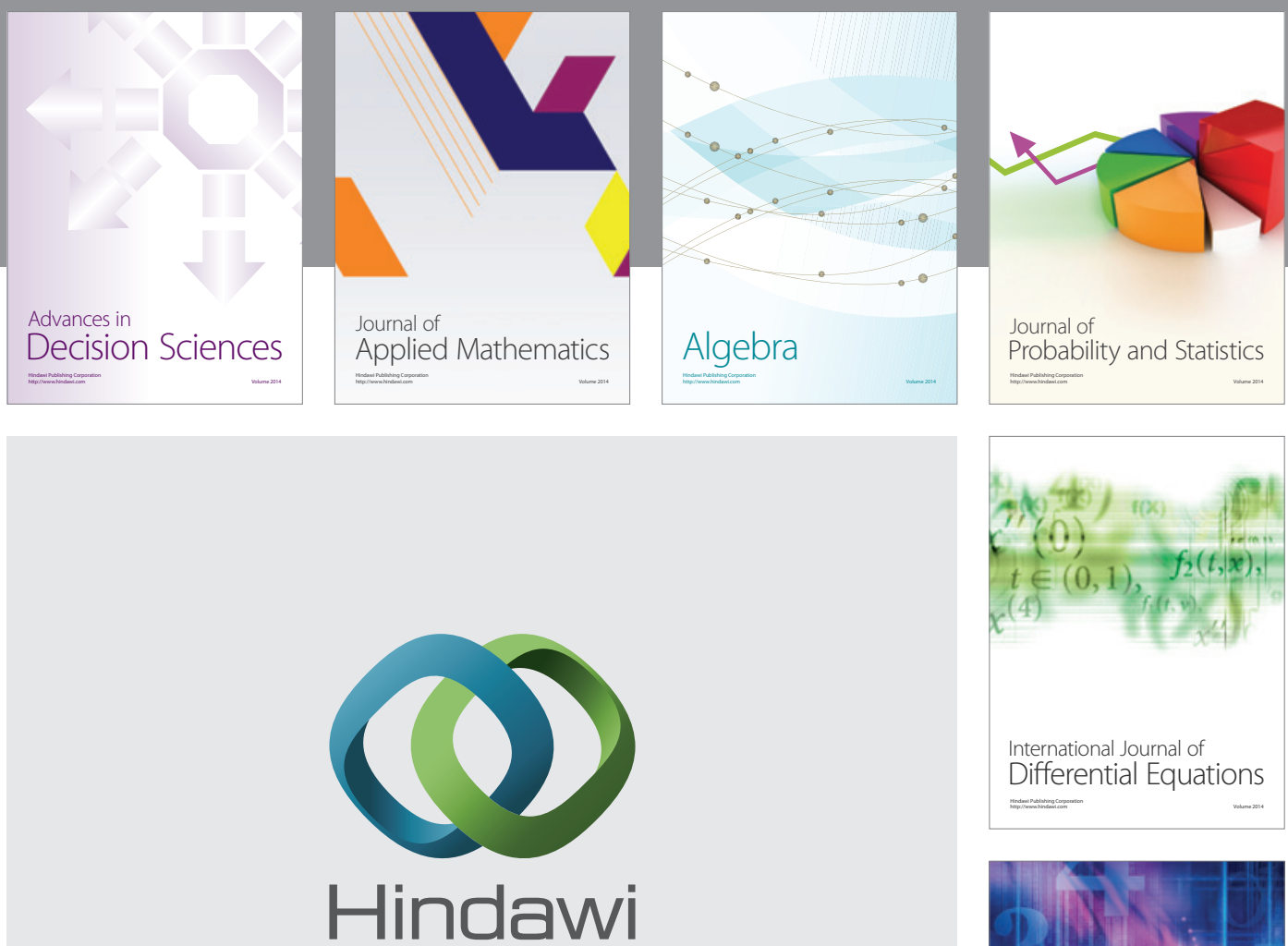

Submit your manuscripts at http://www.hindawi.com
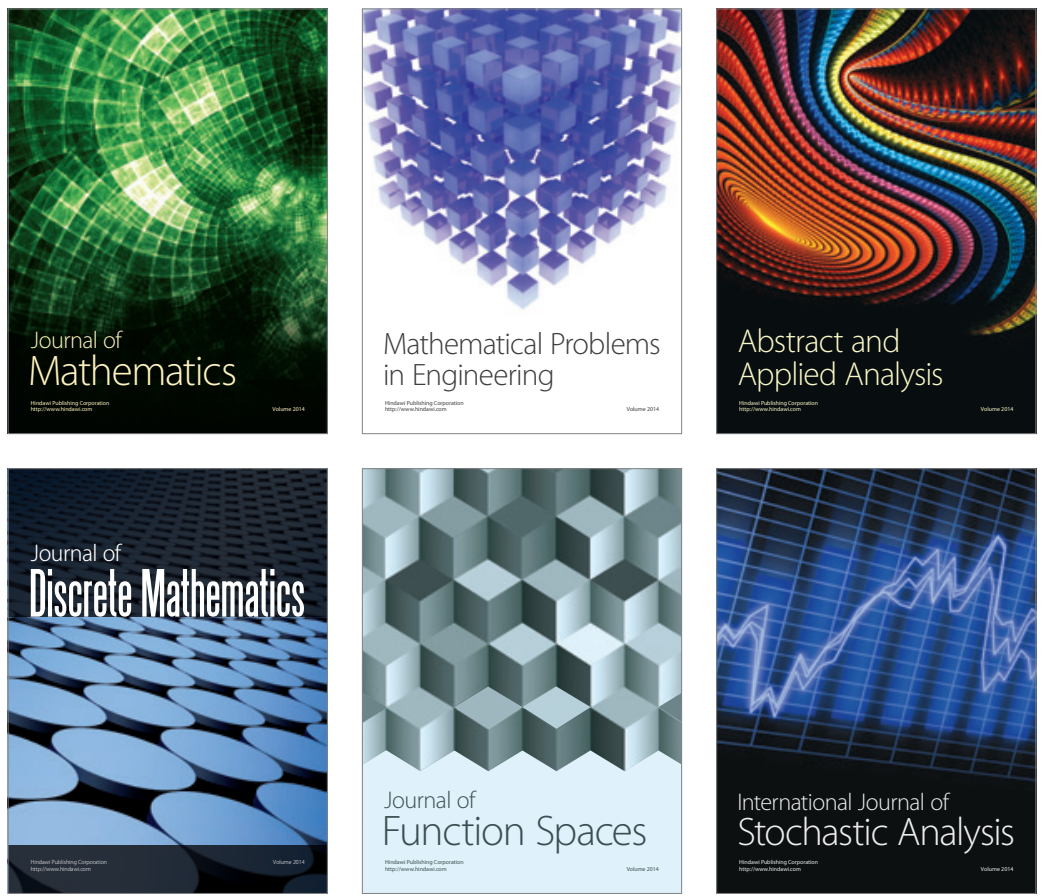

Journal of

Function Spaces

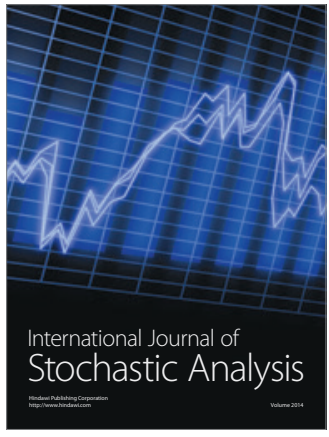

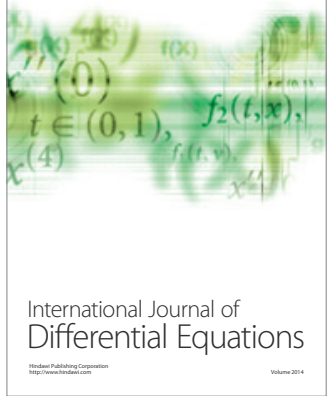
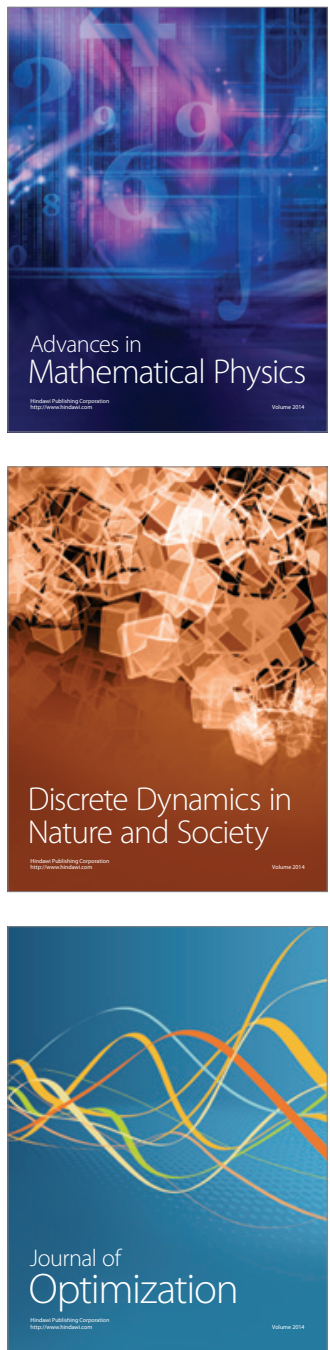